\title{
Work space optimization of a r-r planar manipulator using particle swarm optimization technique
}

\author{
G. Chaitanya ${ }^{1^{*}}$, B. Muddu Krishna ${ }^{2}$ \\ 1*Department of Mechanical Engineering, R.V.R \&J.C College of Engineering, Guntur-19 (A.P), INDIA \\ 2 Department of Mechanical Engineering, R.V.R \&J.C College of Engineering, Guntur-19 (A.P), INDIA \\ ${ }^{*}$ Corresponding Author ${ }^{(1)}$ E-Mail:chaitanyagoteti16@gmail.com
}

\begin{abstract}
A two link revolute planar robotic manipulator is optimized for maximization of work space covered by its end effector. A mathematical model for optimization is built considering singularities which control the range of design variables. Condition number which is the measure of change in output value (End effector position) for a small change in input value (joint angles) is modeled as the constraint. The non linear optimization model is initially linearised using Sequential Linear Programming technique and is solved graphically for optimum value of objective function. Particle swarm optimization technique is implemented on the non-linear optimization model for optimum value of objective function. The maximum value of objective function obtained from Particle swarm technique is found to be significantly higher than the value obtained using graphical approach.
\end{abstract}

Keywords: Singularities, Condition number, Work-space.

DOI: http://dx.doi.org/10.4314/ijest.v9i1.4

\section{Introduction}

It is always desirable to have maximum reach for the robotic end effectors in their work space. However, their movements are restricted by kinematic singularities. It is a well known fact that the Jacobian relates linear velocities of links or end effectors to their joint velocities. At singular positions, the determinant of jacobian becomes zero resulting in infinite joint velocities. Therefore, within these restrictions (singularities), it is area of interest of many researchers to find the global optimum which gives maximum reach for the end effector. Morecki et al. (1984) discussed the effect of link length ratios on the distance travelled by end effector. Yoshikawa (1985) proposed an index which represents the degree of dexterity or manipulability of robotic arm in its work space. It is called manipulability index denoted by $\mu$, where $\mu=\operatorname{detJ}$. Huang and Thebert (2010) made a detailed study on work space singularities for design of parallel robots. Tsai and Chiou (1990) discussed various orientation patterns for joints which result in minimal singularity occurrences. Klein and Blaho (1987) used parameters such as condition number and range of design variables for design and kinematic control of redundant robotic manipulators.

Tokhi and Azad (2008) discussed various strategies for modeling and kinematic design of flexible manipulators. Ata (2006) discussed and presented in detail, various control strategies for design of serial link robotic manipulators. Moldoveanu et al. (2005) presented the variable structure theory for planning and trajectory control of planar two link manipulators. In the present work, the constraint (condition number) is expressed in the form of Euclidean or Frobenious norm of the matrix. The design variables chosen are link lengths and link angle. The objective function and constraint are modeled as nonlinear functions of design variables. Rao (2009) presented the mathematical modeling of various physical problems including mechanical design problems and their optimization methods. For achieving global optimum point, heuristic or evolutionary optimization techniques such as genetic algorithm, particle swarm optimization technique, neural networks, etc., are preferred over conventional optimization techniques as most of these methods deal with a population of vectors. The likelihood of achieving global optimum is very high when working with a population of design vectors simultaneously compared to a single design vector. 
Particle swarm optimization is a heuristic search algorithm based on the concept of sharing and brimming in the population of design vectors. It is similar to the genetic algorithm in the sense that they are both population-based search approaches and they rely on information sharing within the population to improve their search. However, particle swarm optimization uses less number of function evaluations compared to genetic algorithm. The results available from literature support the above claim. Statistical tests were conducted to examine the effectiveness and efficiency of PSO compared to GA. Eight sample test problems were solved using both PSO and GA for multiple runs. The test problems included three popular benchmark problems like Rosen brock function, Egg crate Function, and Golinski's Speed Reducer. The second set of test problems chosen from literature involved the reliability-based design of a commercial communication satellite. All test problems were with continuous design variables.

Two metrics were chosen for the tests. The first metric was to test the effectiveness of PSO and GA using a standard solution as metric that measures the normalized difference between the solutions obtained from the two approaches. The efficiency test tested the number of function evaluations required by each technique to reach convergence. The results of tests showed that though both PSO and GA are similar in terms of quality index or effectiveness to most test problems, it is the PSO technique that showed better rate of convergence in less number of iterations. This clearly indicated high efficiency level of particle swarm optimization technique compared to genetic algorithm. Kennedy and Elberhart initially conceptualized the idea of particle swarm optimization technique based on the social behavior of animals and birds flocking. Later, Bratton and Kennedy (2007) standardized the particle swarm optimization technique. Lee and Park (20006) studied the relative merits and de-merits of applying particle swarm optimization technique to economic dispatch problem. Tasgetiren and Liang (2003) implemented the binary particle swarm optimization technique to determine the economic lot size. Kumar et al (2008) presented in detail, the basic concepts of particle swarm optimization, its variants and its application with reference to power systems. The formulated objective function and constraint are sequentially linearised and the linear problem is solved using graphical approach. Particle swarm optimization technique with 20 swarm values at each iteration is implemented. The maximum value of objective function is found to be $57.5 \%$ higher than the value obtained from graphical method.

\section{Particle Swarm Optimization Technique}

The Particle Swarm Optimization (PSO) method is built based on multiple element parallel search techniques which preserve a flock (swarm) of particles and each particle represents a possible solution in the group. All particles move through an ndimensional search space where each particle will adjust its position based on its previous condition or situation and that of its neighboring particles. In Particle Swarm Optimization technique, all particles are arbitrarily instigated and their fitness is computed to determine local best (best value of each particle) and global best (best value of particle in the entire flock). In the loop, first the particles velocity is updated by the personal and global bests, and then each particle's position is updated by the current velocity. Then the loop will find an optimum solution. The loop will end based on a stopping criterion. Swarm size is the number of particles in the flock or group. Large swarm size creates bigger search space to be covered per iteration. Large number of particles per swarm or flock may reduce the number of iterations for obtaining good result. On the other hand, enormous amount of particles will increase computational time and complexity per iteration. From a number of studies, it is found that, for all practical purposes, the ideal range of swarm size is 10 to 50 .

\section{Mathematical modeling}

Figure 1 shows a schematic representation of a two link planar manipulator with link lengths and link angles.

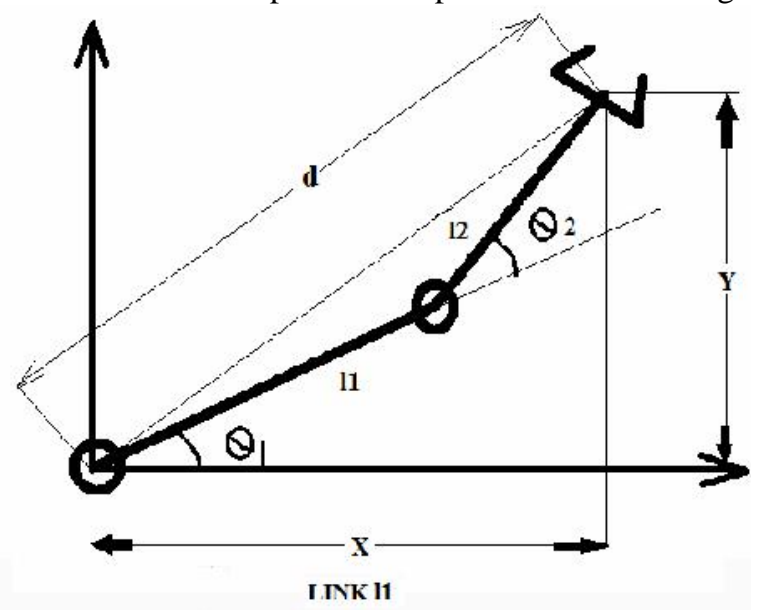

Fig. 1. Two link planar robotic manipulator. 
From forward kinematics we have the following relations connecting end effector positions with joint angles and link lengths.

$$
\begin{aligned}
& x=l_{1} \cos \theta_{1}+l_{2} \cos \left(\theta_{1}+\theta_{2}\right) \\
& y=l_{1} \sin \theta_{1}+l_{2} \sin \left(\theta_{1}+\theta_{2}\right) \\
& d=\sqrt{\left(x^{2}+y^{2}\right)} \\
& d=\sqrt{\left(l_{1}^{2}+l_{2}^{2}+2 l_{1} l_{2} \cos \theta_{2}\right)}
\end{aligned}
$$

Jacobian relates linear velocities of links to joint angular velocities as given below.

$$
\begin{aligned}
& \left(\begin{array}{c}
\dot{X} \\
\dot{Y}
\end{array}\right)=J\left(\begin{array}{c}
\dot{\theta_{1}} \\
\dot{\theta_{2}}
\end{array}\right)=\left(\begin{array}{ll}
\frac{\partial X}{\partial \theta_{1}} & \frac{\partial X}{\partial \theta_{2}} \\
\frac{\partial Y}{\partial \theta_{1}} & \frac{\partial Y}{\partial \theta_{2}}
\end{array}\right)\left(\begin{array}{c}
\dot{\theta_{1}} \\
\dot{\theta_{2}}
\end{array}\right) \text { Where, } \mathrm{J} \text { is the } 2 \mathrm{x} 2 \text { jacobian matrix. } \\
& \left(\begin{array}{c}
\dot{X} \\
\dot{Y}
\end{array}\right)=\left(\begin{array}{ll}
-l_{1} \sin \theta_{1}-l_{2} \sin \left(\theta_{1}+\theta_{2}\right) & -l_{2} \sin \left(\theta_{1}+\theta_{2}\right) \\
l_{1} \cos \theta_{1}+l_{2} \cos \left(\theta_{1}+\theta_{2}\right) & l_{2} \cos \left(\theta_{1}+\theta_{2}\right)
\end{array}\right)\left(\begin{array}{c}
\dot{\theta_{1}} \\
\dot{\theta_{2}}
\end{array}\right)
\end{aligned}
$$

The objective function is modeled keeping in view the extreme positions of end effector or link 2 with respect to link 1 . That is, when $\theta_{2}$ is zero and when $\theta_{2}$ is $180^{\circ}$. At these positions, kinematic or boundary singularities will arise resulting in infinite joint velocities as the determinant of $\mathbf{J}$ will become zero at these two positions. Therefore, the area maneuvered by end effector is obtained by subtracting area when $\theta_{2}$ of link 2 is at $180^{\circ}$ position with respect to link1 from the area when link 2 is zero degrees position with respect to link1. Mathematically:

$$
\text { Area covered by End effector } f\left(l_{1}, l_{2}\right)=\pi\left[\left(l_{1}+l_{2}\right)^{2}-\left(l_{1}-l_{2}\right)^{2}\right]
$$

Equal link length ratio may appear to give maximum work space covered by end effector, but is not Actually the case. Morecki et al. (1984) presented the following Figure 2 in their work which relates the distance travelled by the end effector to link length ratio.

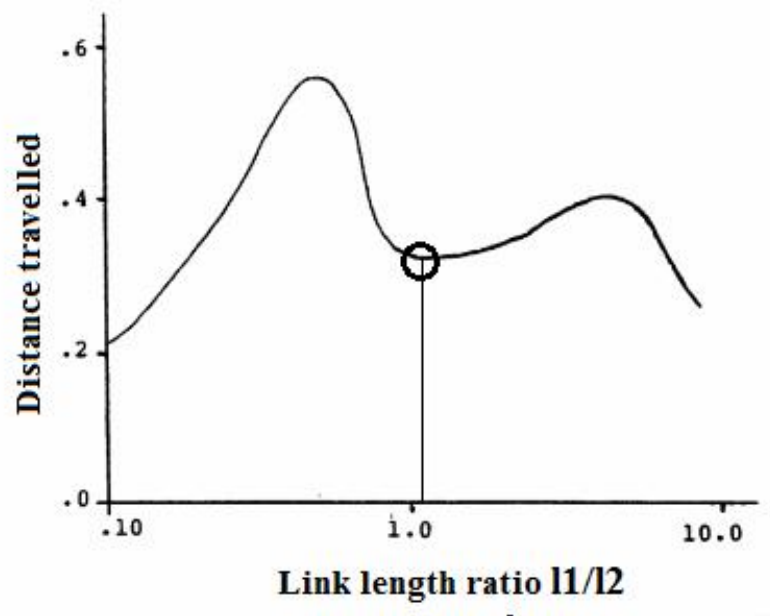

Fig. 2. Distance travelled by end effector with respect to link length ratio

The following link length ratios and ranges of design variables $1_{1}, l_{2}$ and $\theta_{2}$ are arbitrarily chosen keeping in view, the kinematic singularities and propositions made by Morecki et al. (1984).

$$
\frac{l_{1}}{l_{2}} \geq 1.2 \text { and } \frac{l_{1}}{l_{2}} \leq 2
$$

$$
0.1 \leq l_{1} \leq 2 \text { and } 0.1 \leq l_{2} \leq 2,1.832^{c} \leq \theta_{2} \leq 2.705^{c}
$$


Condition number is the error amplification factor, which is defined as the ratio of maximum singularity value to minimum singularity value of a jacobian matrix. The condition number $\mathrm{K}_{\mathrm{J}}$ varies in the range 1 to $\infty$. Condition number close to 1 gives ideal jacobian with almost nil singularities. Condition number close to $\infty$ represents most ill conditioned jacobian matrix for design of manipulator. For the present problem, the upper limit of condition number is chosen equal to 1.25 . Condition number $=K_{J}=\frac{S_{\max }}{S_{\min }}$. The expression for condition number which is the constraint for the present problem can be expressed in terms of design variables using the following matrix analogy. Consider the following matrix:

$$
\begin{aligned}
& P x=q \\
& P(x+\delta x)=q+\delta q
\end{aligned}
$$

Double lines are a representation of Euclidean norm, to distinguish it from regular modulus.

Let $\frac{\|\delta x\|}{\|x\|}$ is the relative error in the solution produced by amplifying relative error in the data $\frac{\|\delta q\|}{\|q\|}$ which is the definition of condition number.

$$
\text { That is } \frac{\|\delta x\|}{\|x\|} \leq K_{J} \frac{\|\delta q\|}{\|q\|}
$$

$P(\delta x)=\delta q \quad$ From equation (b)

$$
\delta x=P^{-1} \delta q
$$

The norm of the matrix is defined as its amplifying power which is expressed as:

$$
\|P x\|=\|P\|\|x\|=\|q\|
$$

Similarly from equation $\mathrm{d}$ we have

$$
\|\delta x\|=\left\|P^{-1} \delta q\right\|=\left\|P^{-1}\right\|\|\delta q\|
$$

Substituting equations e and $\mathrm{f}$ in equation $\mathrm{c}$ :

$$
\frac{\left\|P^{-1}\right\|\|\boldsymbol{\delta} q\|}{\|x\|} \leq K_{J} \frac{\|\boldsymbol{\delta} q\|}{\|P\|\|x\|}
$$

From the above expression, the upper bound for error amplification ratio is written as:

$$
K_{J}=\|P\|\left\|P^{-1}\right\|
$$

Where the norm of matrix $\|P\|$ is the Euclidean or Frobenious norm which is expressed as the square root of sum of the squares of all the elements of matrix P. The same also holds good for matrix $\left\|P^{-1}\right\|$. Mathematically, Euclidean norm may be expressed as follows:

$$
\|P\|=\sqrt{\left(\sum_{i=1}^{m} \sum_{j=1}^{n}\left|P_{i j}\right|^{2}\right)}
$$

Following the above analogy, we have for the present problem $K_{J}=\|J\|\left\|J^{-1}\right\|$. 
Where $\|J\|$ is $\left\|\begin{array}{ll}-l_{1} \sin \theta_{1}-l_{2} \sin \left(\theta_{1}+\theta_{2}\right) & -l_{2} \sin \left(\theta_{1}+\theta_{2}\right) \\ l_{1} \cos \theta_{1}+l_{2} \cos \left(\theta_{1}+\theta_{2}\right) & l_{2} \cos \left(\theta_{1}+\theta_{2}\right)\end{array}\right\|=\sqrt{\left(l_{1}^{2}+2 l_{2}^{2}+2 l_{1} l_{2} \cos \theta_{2}\right)}$

Similarly, $\left\|J^{-1}\right\|=\| \begin{array}{cc}\frac{l_{2} \cos \left(\theta_{1}+\theta_{2}\right)}{l_{1} l_{2} \sin \theta_{2}} & \frac{l_{2} \sin \left(\theta_{1}+\theta_{2}\right)}{l_{1} l_{2} \sin \theta_{2}} \\ \frac{-l_{1} \cos \theta_{1}-l_{2} \cos \left(\theta_{1}+\theta_{2}\right)}{l_{1} l_{2} \sin \theta_{2}} & \frac{-l_{1} \sin \theta_{1}-l_{2} \sin \left(\theta_{1}+\theta_{2}\right)}{l_{1} l_{2} \sin \theta_{2}}\end{array}$

$$
\left\|J^{-1}\right\|=\frac{\sqrt{\left(l_{1}^{2}+2 l_{2}^{2}+2 l_{1} l_{2} \cos \theta_{2}\right)}}{l_{1} l_{2} \sin \theta_{2}}
$$

Therefore, the expression for condition number is given as shown below:

$$
K_{J}=\frac{\left(l_{1}^{2}+2 l_{2}^{2}+2 l_{1} l_{2} \cos \theta_{2}\right)}{l_{1} l_{2} \sin \theta_{2}}
$$

Therefore, the optimization problem for the two link revolute planar manipulator can be represented as follows from equations 5 to 8. $\min (\mathrm{f})=-\pi\left[\left(l_{1}+l_{2}\right)^{2}-\left(l_{1}-l_{2}\right)^{2}\right]$ Minimizing negative of a function results in maximization.

s.t

$l_{1}-1.2 l_{2} \geq 0$

$l_{1}-2 l_{2} \leq 0$

$K_{J} \equiv\left(l_{1}^{2}+2 l_{2}^{2}+2 l_{1} l_{2} \cos \theta_{2}-1.25 l_{1} l_{2} \sin \theta_{2}\right) \leq 0$

for

$0.2 \leq l_{1} \leq 2$

$0.2 \leq l_{2} \leq 2$

$1.832^{c} \leq \theta_{2} \leq 2.705^{c}$

\section{Sequential Linear programming}

The objective function and constraint are linearised using the following relations with a initial starting feasible solution $X^{*}$.

$$
\begin{aligned}
& f_{1}(X)=f\left(X^{*}\right)+\nabla f\left(X^{*}\right)^{T}\left(X-X^{*}\right) \\
& g_{1}(X)=g\left(X^{*}\right)+\nabla g\left(X^{*}\right)^{T}\left(X-X^{*}\right) \\
& \text { where } X^{*}=\left[\begin{array}{l}
l_{1} \\
l_{2} \\
\theta_{2}
\end{array}\right]=\left[\begin{array}{c}
0.5 \\
0.25 \\
1.832^{c}
\end{array}\right] \text { is the initial feasible solution chosen. }
\end{aligned}
$$

The linear programming problem after first sequence of linearization with starting solution is:

$$
\min f_{1}\left(l_{1}, l_{2}\right)=-\pi l_{1}-\pi l_{2}+0.7854
$$




$$
\begin{aligned}
& \text { s.t } \\
& g_{1}\left(l_{1}, l_{2}, \theta_{2}\right)=1.172 l_{1}+1.345 l_{2}-0.282 \theta_{2}-0.246 \leq 0 \\
& g_{2}\left(l_{1}, l_{2}\right)=-l_{1}+1.2 l_{2} \leq 0 \\
& g_{3}\left(l_{1}, l_{2}\right)=l_{1}-2 l_{2} \leq 0 \\
& \text { for } \\
& 0.2 \leq l_{1} \leq 2 \\
& 0.2 \leq l_{2} \leq 2 \\
& 1.832^{c} \leq \theta_{2} \leq 2.705^{c}
\end{aligned}
$$

The problem is subsequently linearised by taking the most violated constraint which is obtained by substituting the optimal solution obtained at the end of each LPP run in the original nonlinear problem. Since, the graphical method uses only two variables, the problem is solved for $l_{1}$ and $l_{2}$ by varying the value of $\theta_{2}$ by a factor of $0.175^{\mathrm{c}}$ in constraint $\mathrm{g}_{1}$ starting from $1.832^{\mathrm{c}}$ for each iteration. The following figure 3 shows the final optimal values of link lengths. The maximum value of objective function is found to be 19.1 square units at $l_{1}=0.643$ units and $l_{2}=0.585$ units for $\theta_{2}=135^{\circ}$.

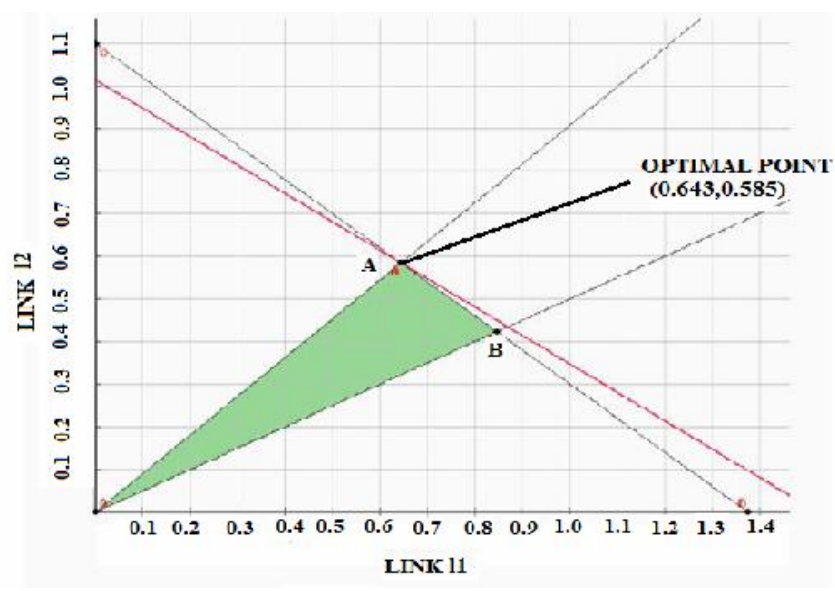

Fig. 3. Optimal values of link lengths

\section{Particle Swarm Optimization parameters for the present problem}

The following inputs are chosen to implement the Particle swarm optimization Technique:

No of variable $=3$

No of particles $/$ Iteration $=10$

Maximum number of Epochs $=60$

No of Iterations $=200$

The following equation illustrates the updation of velocities of particles

$$
V^{t+1}=V^{t}+c_{1} r_{1}^{t}\left(p_{\text {best }}-x^{t}\right)+c_{2} r_{2}^{t}\left(G_{\text {best }}-x^{t}\right)
$$

$\mathrm{C}_{1}=1.5, \mathrm{C}_{2}=1.5$ (Learning factors can take any arbitrary values and need not be equal). $\mathrm{r}_{1}$ and $\mathrm{r}_{2}$ are random numbers whose value lies between 0 and $1 . P_{\text {best }}$ or local best is the best improved position of a particular particle from start of iterations. $G_{\text {best }}$ or Global best is best position reached by any of the particles in the entire swarm from the start of iterations. $\mathrm{x}^{\mathrm{t}}$ is the position vector of the particle and $\mathrm{V}^{\mathrm{t}}$ and $\mathrm{V}^{\mathrm{t}+1}$ are velocities before and after updation. The following flow chart shown in Figure 4 illustrates the working of Particle swarm optimization technique. 


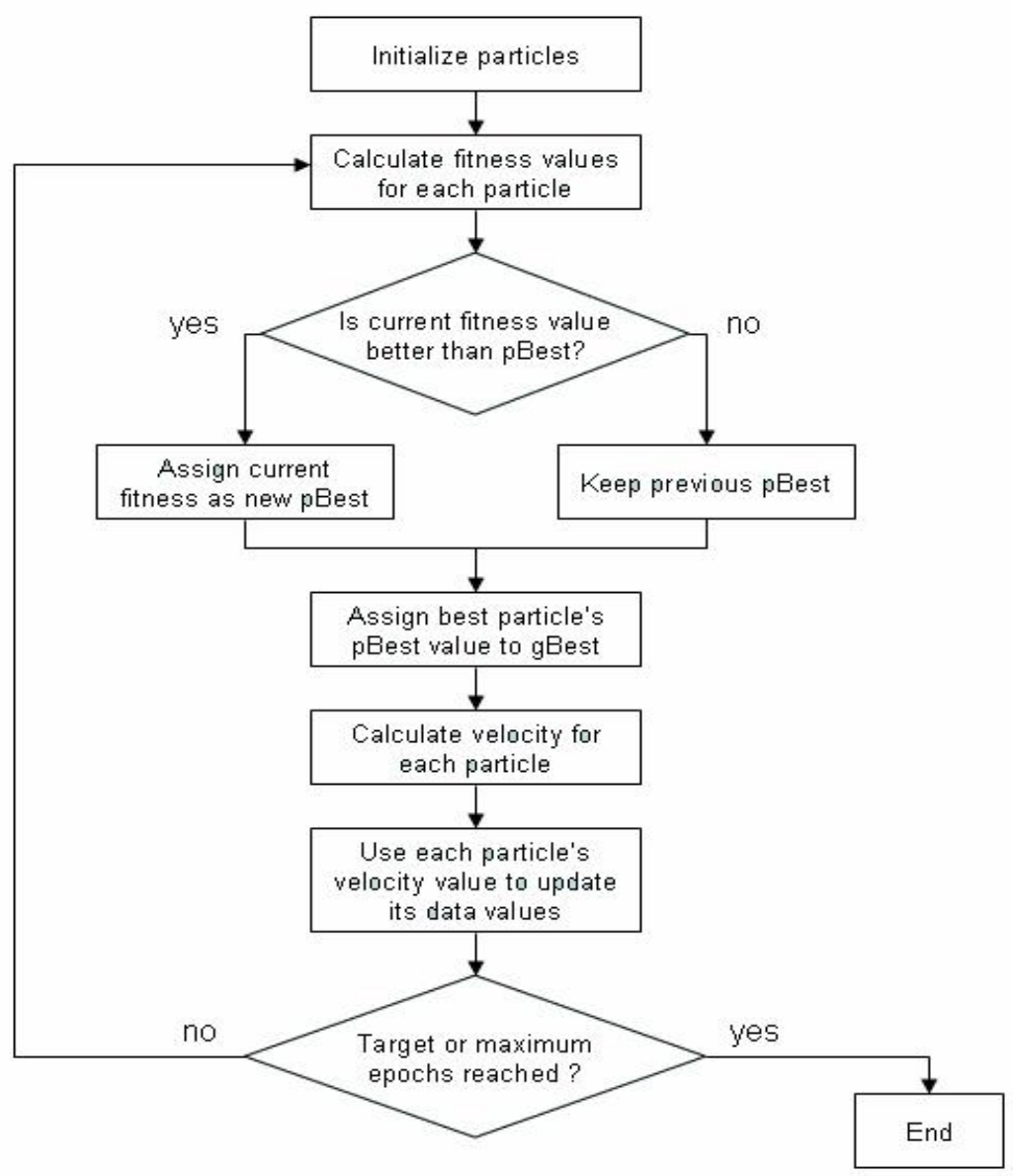

Fig. 4. Particle swarm optimization flow chart

The objective function value versus number of iterations is shown in Figure 5. The maximum value of the objective function obtained from this method is found to be 45.2 square units. The optimal values of design variables are found to be $1_{1}=1.99$ units, $1_{2}=1.8$ units and $\theta_{2}=125.47^{0}$.

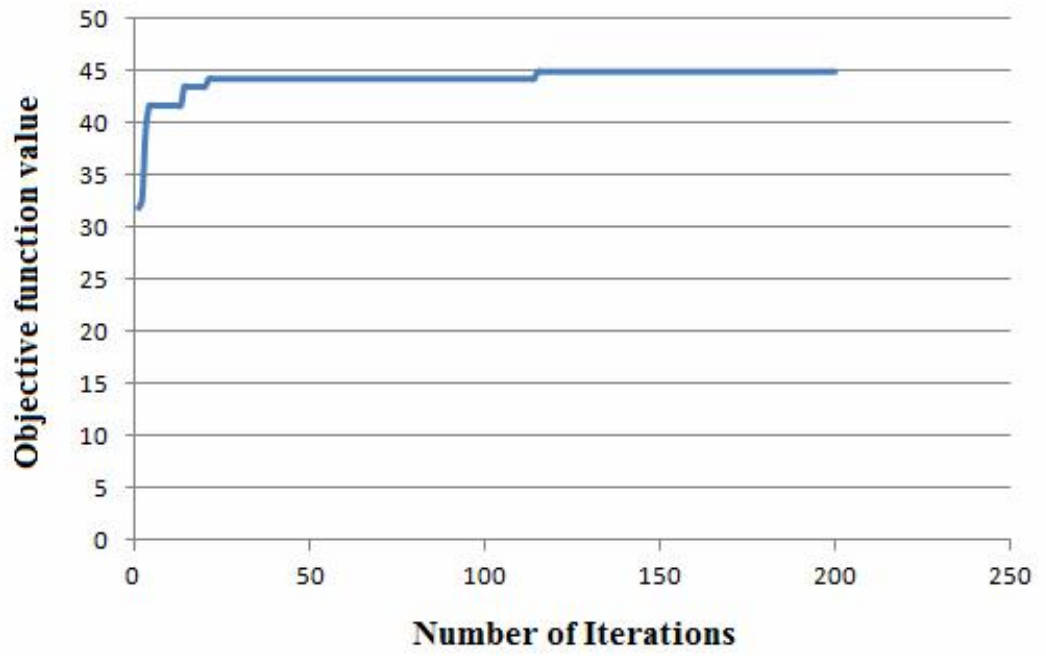

Fig. 5. Objective function value vs. No of Iterations 
Table1: Deviation in objective function value with iterations

\begin{tabular}{|c|c|c|}
\hline Iteration number & Objective function value & Percentage deviation from previous iteration number \\
\hline 10 & 40 & 2 \\
\hline 15 & 41.2 & 2.91 \\
\hline 20 & 41.2 & 0 \\
\hline 25 & 41.2 & 0 \\
\hline 30 & 43.8 & 5.9 \\
\hline 35 & 43.8 & 0 \\
\hline 40 & 44.5 & 1.57 \\
\hline 50 & 44.5 & 0 \\
\hline 125 & 45.2 (optimum) & 1.55 \\
\hline
\end{tabular}

\section{Results-Discussion}

By comparing the objective function value obtained from the two approaches, it can be seen that the value of objective function obtained from particle swarm technique is found to be $57.7 \%$ larger than the value obtained from the graphical approach. This is attributed to the fact that PSO technique uses a swarm or flock of particles, each of which represents a possible solution to the problem at hand and the G-best among those particles has high likely hood of representing global optimum. On the other hand, graphical method used a set of linear equations to find the optimum. This resulted in local optimum. Also, the original nonlinear problem is converted into a linear problem using Sequential linear programming technique and the solution obtained from graphical method satisfied the original nonlinear constraints by a degree given by $\varepsilon$ which is a very small value close to zero. On the other hand PSO worked on the original non linear problem itself which resulted in exact satisfaction of all the constraints. The optimum value of objective function obtained from PSO technique stabilized after $125^{\text {th }}$ iteration and remained constant till the end of $200^{\text {th }}$ iteration indicating global optimum. However, from the results in Table1, it can be observed that the objective function value obtained from PSO technique showed a significant improvement over the value of objective function (19.1 square units) obtained from LPP approach within the first 10 iterations. This analysis would be helpful to assess the optimum value in a situation where fast convergence is required.

\section{Conclusions}

The following major conclusions are drawn from the work:

- The maximum area covered by end effector obtained from PSO technique is found to be $57.7 \%$ higher than the value obtained from Graphical method.

- Link angle $\theta_{2}$ is found to have significant influence on condition number followed by link lengths.

- The choice of learning factors in the present work influenced the convergence rate of PSO algorithm.

\section{References}

Ata A. A. 2006, Optimal trajectory planning of manipulators: A review, Journal of Engineering Science and Technology, Vol. 2, No. 1, pp. 32-54.

Bratton D. and Kennedy J., 2007, Defining a standard for particle swarm optimization, IEEE Swarm Intelligence Symposium, pp. 120-127.

del Valle Y., Venayagamoorthy G.K., Mohagheghi S., Hernandez J.-C., Harley R.G., 2008, Particle swarm optimization: Basic concepts, variants and applications in power systems, IEEE, pp. 171-195.

Huang M.Z. and Thebert J.-L. 2010, A study of workspace and singularity characteristics for design of 3-DoF planar parallel robots, International Journal of Advanced Manufacturing Technology, Vol. 51, No. 5-8, pp. 789-797.

Klein C. A. and Blaho B. E., 1987, Dexterity measures for the design and control of kinematically redundant manipulators, The International Journal of Robotics Research, Vol. 6, No. 2, 1987, pp. 72-83.

Lee K.Y. and Park J.-B., 2006, Application of particle swarm optimization to economic dispatch problem: Advantages and disadvantages, Power Systems Conference and Exposition, 2006. PSCE '06, 29 Oct.-1 Nov. 2006, DOI: 10.1109/PSCE.2006.296295 
Moldoveanu F., Comnac V. and Floroia D. 2005, Trajectory tracking control of a two-link robot manipulator using variable structure system theory, Journal of Control Engineering and Applied Informatics, Vol. 7, pp. 56-62.

Morecki A., Bianchi G. and Kedzior K., 1984, Theory and practice of robots and manipulators, Proceedings of Fifth CISMIFTOMM Symposium, pp. 41-46.

Rao S.S., 2009, Engineering Optimization Theory and Practice, 4th edition, Ed., John Wiley and Sons.

Tasgetiren M.F. and Liang Y.-C., 2003, A binary particle swarm optimization algorithm for lot sizing problem, Journal of Economic and Social Research, Vol. 5, No. 2, pp. 1-20.

Tokhi M.O. and Azad A.K.M., 2008, Flexible Robot Manipulators Modeling, Simulation and Control, IET, London, UK.

Tsai M. J. and Chiou Y. H., 1990, Manipulability of manipulators, Mechanism and Machine Theory, Vol. 25, No. 5, pp. 575-585.

Yoshikawa T., 1985, Manipulability of robotic mechanisms, The International Journal of Robotics Research, Vol. 4, No 2, pp. 39.

\section{Biographical notes}

Dr. G. Chaitanya obtained is PhD in Mechanical Engineering from JNTU Hyderabad in the year 2012. He is presently working as Associate Professor in the Department of Mechanical Engineering at R.V.R\& J.C College of Engineering Guntur, Andhra Pradesh, India. He has over 12 years of teaching experience and published over 20 research articles in various journals and is presently guiding 5 students for $\mathrm{PhD}$.

Mr. B. Muddu Krishna obtained his M.Tech in C.A.D/C.A.M from Vellore Institute of Technology, Vellore and B.Tech in Mechanical Engineering from JNTU Kakinada. He is presently working as Associate Professor in the Department of Mechanical Engineering at R.V.R\&J.C College of Engineering Guntur, Andhra Pradesh, India.

Received July 2016

Accepted March 2017

Final acceptance in revised form April 2017 